\title{
Elevated serum and BAL cytokeratin 19 fragment in pulmonary fibrosis and acute interstitial pneumonia
}

\author{
N. Dobashi*, J. Fujita*, Y. Ohtsuki*, I. Yamadori ${ }^{\S}$, T. Yoshinouchi $^{+}$, T. Kamei ${ }^{\#}$, J. Takahara*
}

\begin{abstract}
Elevated serum and BAL cytokeratin 19 fragment in pulmonary fibrosis and acute interstitial pneumonia. N. Dobashi, J. Fujita, Y. Ohtsuki, I. Yamadori, T. Yoshinouchi, T. Kamei, J. Takahara. C ERS Journals Ltd 1999.

ABSTRACT: Cytokeratin 19 fragment (CK19) levels in serum have already been documented as a useful tumour marker for lung cancer. In the present study, it was hypothesized that CK19 may be increased in the serum and epithelial lining fluid of the respiratory tract from patients with pulmonary fibrosis.

CK19 was measured in the serum and bronchoalveolar lavage fluid (BALF) of patients with pulmonary fibrosis and the correlation between CK19 levels and clinical parameters evaluated. Nineteen patients diagnosed with idiopathic pulmonary fibrosis (IPF), eight with pulmonary fibrosis associated with a collagen vascular disorder (PF-CVD), seven patients with acute interstitial pneumonia (AIP), and 10 normal smokers as a control group were studied. CK19 levels in sera of patients with IPF and patients with PF-CVD were significantly increased compared to those of normal smokers.
\end{abstract}

CK19 levels in sera of patients with AIP were significantly increased compared to those of other groups. CK19 values in the BALF of patients with pulmonary fibrosis were significantly elevated compared to those of normal smokers. CK19 values in sera charged according to the progression or improvement of the acute lung injury. Immunohistochemical study using pulmonary tissues obtained from patients with AIP demonstrated that the hyaline membrane and proliferating type II pneumocytes were stained by anti-human cytokeratin 19 antibody.

These data demonstrated that the measurement of cytokeratin 19 fragment is a useful parameter to evaluate the activity of lung epithelial cell damage and repair. Eur Respir J 1999; 14: 574-578.
*First Dept of Internal Medicine, Kagawa Medical University, Kagawa, Japan. ${ }^{+}$Dept of Respiratory Medicine, Gamagohri City Hospital, Ehime, Japan. "Dept of Internal Medicine, Kagawa Prefectural Central Hospital, Kagawa, Japan. ${ }^{8}$ Dept of Pathology, Okayama University Medical School, Okayama, Japan. "Dept of Pathology, Kochi Medical School, Kochi, Japan.

Correspondence: J. Fujita, First Dept of Internal Medicine, Kagawa Medical University, 1750-1 Miki-cho, Kita-gun, Kagawa 761-0793, Japan. Fax: 1181878912147

Keywords: Acute interstitial pneumonia collagen vascular disorder cytokeratin 19 fragment hyaline membrane idiopathic pulmonary fibrosis

Received: February 171998 Accepted after revision April 221999
Cytokeratin 19 fragment (CK19) is a specific cytoskeletal structure of simple epithelia, including bronchial epithelial cells [1-4].

Although the precise mechanisms of their synthesis are unknown, fragments of cytokeratin are soluble in serum and can be detected with the aid of monoclonal antibodies in patients with lung cancer [5-8]. In addition, there are few reports which evaluate CK19 levels in serum and bronchoalveolar lavage fluid (BALF) in interstitial lung diseases $[9,10]$. Since it has been suggested that CK19 is released from injured bronchial epithelium [11], it was hypothesized that the levels of CK19 in serum and BALF may also be increased in patients with pulmonary fibrosis, and that the levels measured may correlate with other clinical and biochemical parameters. On this basis, the CK19 levels in serum from patients with idiopathic pulmonary fibrosis (IPF), patients with pulmonary fibrosis associated with collagen vascular disorders (PF-CVD), and patients with acute interstitial pneumonia (AIP) were measured. CK19 levels in BALF were also measured in patients with IPF and PF-CVD.

\section{Materials and methods}

\section{Subjects}

The protocols of this study were approved by the institutional review board for human studies and inform- ed written consent was obtained from the subjects. Twenty-seven patients from Kagawa Medical University Hospital (613 beds) and Matsuyama-Shimin Hospital (538 beds) diagnosed with pulmonary fibrosis were studied during 1990-1996. Of the 27 patients, 19 had IPF and eight had PF-CVD (two had rheumatoid arthritis, five dermatomyositis, and one had Sjögren syndrome). The median (range) age of the patients with IPF was 68 yrs (range 44-86 yrs) (11 males, 8 females). There were 12 nonsmokers, two exsmokers, and seven current smokers. Median (range) age of the patients with PF-CVD was 55 yrs range (30-67 yrs) (two males, six females). There were six nonsmokers and two current smokers. The diagnosis of pulmonary fibrosis was made by clinical, radiological, physiological and histological grounds. The criteria used included: history of exertional dyspnoea end cough, fine crackles on physical examination, compatible findings on the chest radiograph (diffuse basal reticulonodular shadowing), physiological abnormalities of restrictive lung defects including decreased diffusing capacity, and abnormal arterial oxygen tension $\left(\mathrm{Pa}_{\mathrm{a}} \mathrm{O}_{2}\right)$ at rest and/or with exertion. Histological confirmation was obtained in all cases by open lung biopsy. The histology of all patients with PF-CVD had the pattern of usual interstitial pneumonia (UIP). In all patients, high resolution computed tomography (HRCT) scanning of the lungs was performed. All patients received no immunosuppressive treatment 
such as corticosteroid or cyclophosphamide at the time of open lung biopsy.

CK19 was also measured in seven patients with AIP (median age 61 yrs, four males, three females). All patients fulfilled the clinical and pathologic criteria of AIP as described by KATZENSTEIN et al. [12]. Thus, all patients presented with symptoms of acute respiratory failure and had pathological findings of diffuse alveolar damage with thickening of the alveolar walls due to oedema, inflammatory cells and active fibroblast proliferation. None of the patients was at risk for adult respiratory distress syndrome. Among seven patients, four patients died of respiratory failure.

Ten normal smokers (smoking $\geq 1$ pack $\cdot$ day $^{-1}$, nine males, one female) average age $45 \mathrm{yrs}$, were also studied. All subjects had no history of lung diseases, no clinical findings suggesting lung disease and normal liver and renal function. They all had normal chest radiographs and their pulmonary function test results were within a normal range.

\section{Blood samples}

Peripheral venous blood samples with and without ethylenediaminetetraacetic acid (EDTA) were obtained at 07:00 $\mathrm{h}$ before breakfast. After centrifugation at 1,000 $\times \mathrm{g}$ for $10 \mathrm{~min}$ at $4{ }^{\circ} \mathrm{C}$, the serum was frozen and stored at $-70^{\circ} \mathrm{C}$ until used. Arterial blood samples were analysed for $P \mathrm{a}, \mathrm{O}_{2}$ and arterial carbon dioxide tension $\left(\mathrm{Pa}_{\mathrm{a}}, \mathrm{CO}_{2}\right)$ using a blood gas analyser (Ciba Corning 840; Ciba Corning Diagnostics, Tokyo, Japan).

\section{Bronchoalveolar lavage}

After the upper airway was anaesthetized with topical lidocaine $(1 \%$, total $10 \mathrm{~mL})$, a flexible fibreoptic bronchoscope (Type 30; Olympus Co., Tokyo, Japan) was wedged into the lower respiratory tract under visual control. To sample the lower respiratory tract, BAL was performed by infusing three $50 \mathrm{~mL}$ aliquots of sterile saline at the site of the anterior segment of the right lower lobe. The final two aliquots were preserved for evaluation. The fluid was filtered through sterile gauze $(0.5 \times 0.5 \mathrm{~mm}$; Hakuzo Medical Co., Osaka, Japan), and cells were separated from the alveolar lavage fluid by centrifugation $\left(300 \times g\right.$ for $10 \mathrm{~min}$ at $\left.24^{\circ} \mathrm{C}\right)$. BALF was frozen and stored at $-70^{\circ} \mathrm{C}$ until used.

Measurement of cytokeratin 19 fragment levels in serum and bronchoalveolar lavage fluid

CK19 levels in BALF were measured in patients in whom BAL could be performed. CK19 in serum and BALF were measured with a two-step sandwich immunoassay using the streptavidin-biotin technique (EnzymunTest CYFRA21-1; Boehringer Mannheim GmbH, Tutzing, Germany). The lower detection limit of this assay was 0.1 $\mathrm{ng} \cdot \mathrm{mL}^{-1}$. Data were expressed as mean values from duplicate measurements.

In patients with pulmonary fibrosis, correlations between CK19 levels and other parameters were evaluated by measuring the following. In serum: CK19, elastase: $\alpha 1-$ proteinase inhibitor complex (E-PI), hepatocyte growth factor (HGF), C-reactive protein (CRP), lactate dehydro- genase (LDH) and immunoglobulin $(\mathrm{Ig}) \mathrm{G}$; in BALF: CK19, E-PI, HGF, LDH, IgG, IgA, tumour markers (carcinoembryonic antigen (CEA), the carbohydrate antigen sialyl Lewis (a) (CA19-9), squamous cell carcinoma antigen (SCC)), albumin and cell differentials (\% macrophage, $\%$ neutrophils and \% lymphocytes etc.); in peripheral venous blood: white blood cell numbers (WBC) and the erythrocyte sedimentation rate (ESR). Arterial blood gas tensions $\left(\mathrm{Pa}_{\mathrm{a}} \mathrm{O}_{2}\right.$ and $\left.\mathrm{Pa}, \mathrm{CO}_{2}\right)$ were also measured. In addition, as pulmonary function tests, per cent vital capacity $(\% \mathrm{VC})$ and forced expiratory volume in one second (FEV1) were measured.

The E-PI concentration was determined using enzymelinked immunosorbent assay (ELISA) kits (Diagnostica Merk, Darmstadt, Germany). HGF was measured by ELISA with monoclonal and polyclonal antibodies against human HGF (Otsuka Assay Laboratories, Tokushima, Japan). LDH in BALF was measured as the enzyme activity. Tumour markers were measured by radioimmunoassay. Albuminin BALF was measured by ELISA (Albuwell; Exocell Inc., Philadelphia, PA, USA). Data in BALF were corrected to albumin.

\section{Immunchistochemical staining}

Immunohistochemical staining was performed by the avidin-biotin peroxidase complex method (Dako LSAB kit-peroxidase, DAKO Corp., Kyoto, Japan). Monoclonal antibody to CK19 was purchased from ScyTek Laboratories (Logan, UT, USA) and used at 1:30 dilution. In order to enhance the immune reaction, dewaxed sections were pretreated in an autoclave at $132^{\circ} \mathrm{C}$ for $12 \mathrm{~min}$ in $10 \mathrm{mM}$ citrate buffer ( $\mathrm{pH}$ 6.0). Staining procedures were performed according to the manufacturers' instructions.

\section{Statistical methods}

Results were expressed as mean $\pm \mathrm{SD}$. Comparisons of values between the two groups were analysed with the Mann-Whitney U-test. Correlations were evaluated by the Pearson's correlation coefficient, and Fisher's $r$ to $z$ method was used to calculate the p-values. A p-value $<0.05$ was considered as significant.

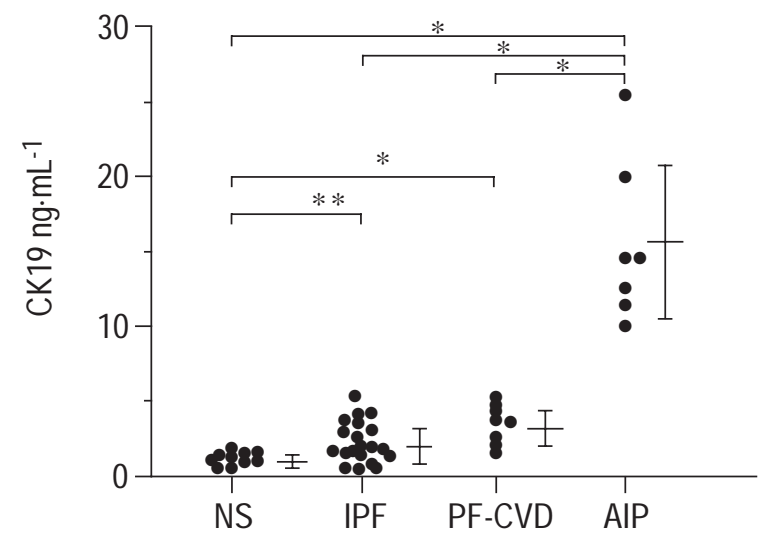

Fig. 1. - Serum cytokeratin 19 fragment (CK19) levels in normal smokers (NS), patients with idiopathic pulmonary fibrosis (IPF), patients with pulmonary fibrosis associated with collagen vascular disorders (PFCVD), and patients with acute interstitial pneumonia (AIP). Bars represent mean \pm SD deviation. *: $\mathrm{p}<0.001 ; * *$ : $\mathrm{p}<0.02$. 


\section{Results}

Figure 1 shows the CK19 levels in serum of normal nonsmokers, patients with IPF, PF-CVD, or AIP. CK19 levels in serum of patients with IPF $\left(2.1 \pm 1.3 \mathrm{ng} \cdot \mathrm{mL}^{-1}\right)$, and patients with PF-CVD $\left(3.2 \pm 1.3 \mathrm{ng} \cdot \mathrm{mL}^{-1}\right)$ were significantly elevated as compared with normal smokers $\left(1.0 \pm 0.4 \mathrm{ng} \cdot \mathrm{mL}^{-1}\right) \quad(\mathrm{p}<0.02$ and $\mathrm{p}<0.001$, respectively $)$. CK19 levels in sera of patients with AIP $(15.4 \pm 5.4$ $\left.\mathrm{ng} \cdot \mathrm{mL}^{-1}\right)$ were significantly increased compared to the other groups $(\mathrm{p}<0.001)$.

Figure 2 shows the CK19 levels in the BALF of patients with pulmonary fibrosis (both IPF and PF-CVD, $\mathrm{n}=10)$ and normal smokers $(\mathrm{n}=10)$. CK19 levels in the BALF of patients with pulmonary fibrosis $(1.57 \pm 1.3$ $\left.\mathrm{ng} \cdot \mathrm{mL}^{-1}\right)$ were significantly high compared with normal smokers $\left(0.37 \pm 0.36 \mathrm{ng} \cdot \mathrm{mL}^{-1}, \mathrm{p}<0.02\right)$.

Figure 3 shows the changes in serum CK19 levels in patients with AIP according to the treatment. Patients were treated by pulse therapy with intravenous methylprednisolone ( $1 \mathrm{~g} \cdot \mathrm{day}^{-1}$ for 3 days) and followed by oral prednisolone $\left(1 \mathrm{mg} \cdot \mathrm{kg}^{-1}\right)$ prednisolone was gradually reduced and continued for at least 6 months. In patients with AIP, CK19 levels increased according to the clinical course. In addition, in patients where the AIP was improved by treatment, CK19 levels decreased according to the clinical course (time interval was 4 weeks). Interestingly, CK19 levels in serum did not correlated with the CRP values in serum (fig. 4).

Table 1 shows the correlation between CK19 in serum and in BALF with several clinical parameters. CK19 levels in sera had a significant negative correlation with $\% \mathrm{VC}(\mathrm{r}=-0.55, \mathrm{p}<0.02)$. CK19 levels in BALF had a significant correlation with several inflammatory cells in BALF and with LDH in BALF. Levels of serum CK19 did not correlate with the levels of E-PI, HGF, CRP, LDH or IgG in serum, nor with the levels of E-PI, HGF, LDH, IgG, CEA, CA19-9 or SCC in BALF. In addition, the levels of CK19 or CK19/albumin ratio in BALF did not correlate with E-PI, HGF, CRP, LDH, or IgG in serum, nor with E-PI, HGF, IgG, CEA, or SCC in BALF.

Immunostaining of hyaline membrane with anti-human CK19 monoclonal antibody was positive in all autopsied

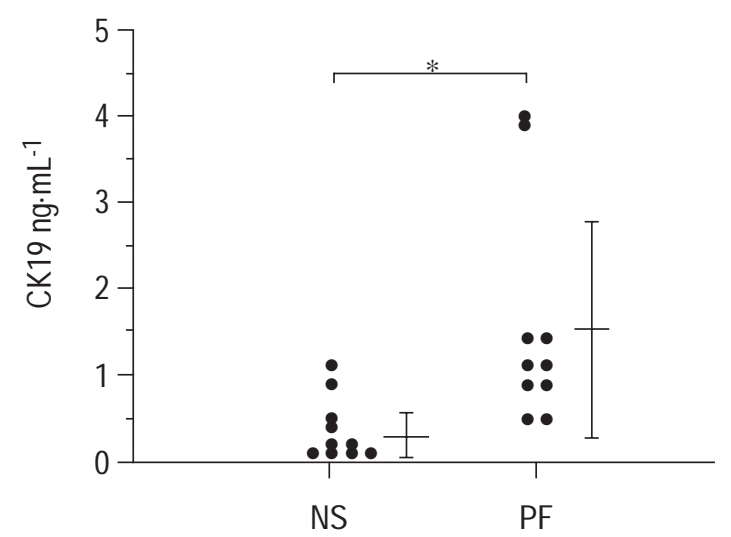

Fig. 2. - Cytokeratin 19 fragment (CK19) levels in the bronchoalveolar lavage fluid (BALF) of normal smokers and patients with pulmonary fibrosis (patients with idiopathic pulmonary fibrosis and pulmonary fibrosis associated with collagen vascular disorders). Bars represent mean \pm SD. ${ }^{*}: \mathrm{p}<0.02$.
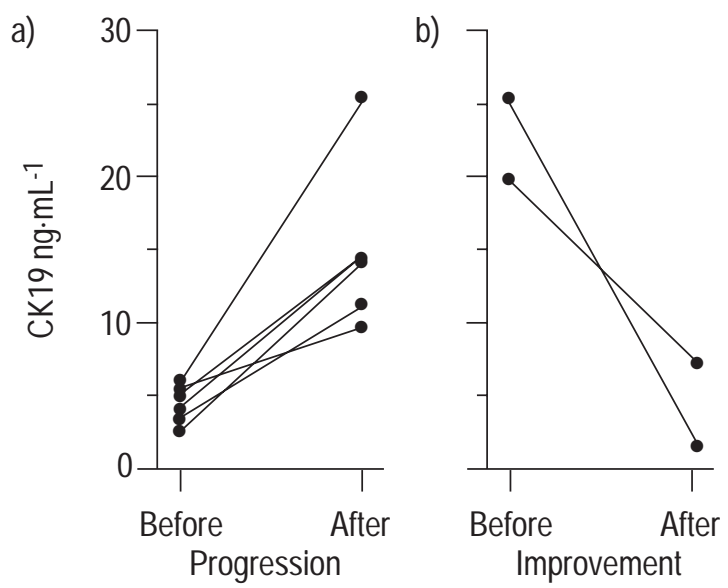

Fig. 3. - Changes of serum cytokeratin 19 fragment (CK19) levels according to clinical courses in seven patients with acute interstitial pneumonia (AIP). a) Progressed cases; b) improved cases. In patients with AIP, CK19 levels increased according to the progressing clinical course (a). In contrast, in patients in which AIP was improved by treatment, CK19 levels decreased according to the improvement clinical course (b)

cases of AIP ( $\mathrm{n}=3$ ) as shown in figure 5 (arrows). Although hyaline membranes stained mostly positive, the density of immunoreactivity was variable in different parts of each case examined. Proliferating type II pneumocytes were stained much more densely than the hyaline membranes, as demonstrated in figure 5 .

\section{Discussion}

In the present study, it was demonstrated that the CK19 levels in serum of patients with pulmonary fibrosis were significantly higher compared with normal smokers, and that CK19 in serum significantly correlated with some clinical parameters in patients with pulmonary fibrosis. In addition, CK19 levels in serum of patients with AIP were extraordinarily high and correlated with the clinical course. Furthermore, immunohistochemical staining demonstrated that the anti-CK19 antibody stained to a moderate density at the site of the hyaline membrane.

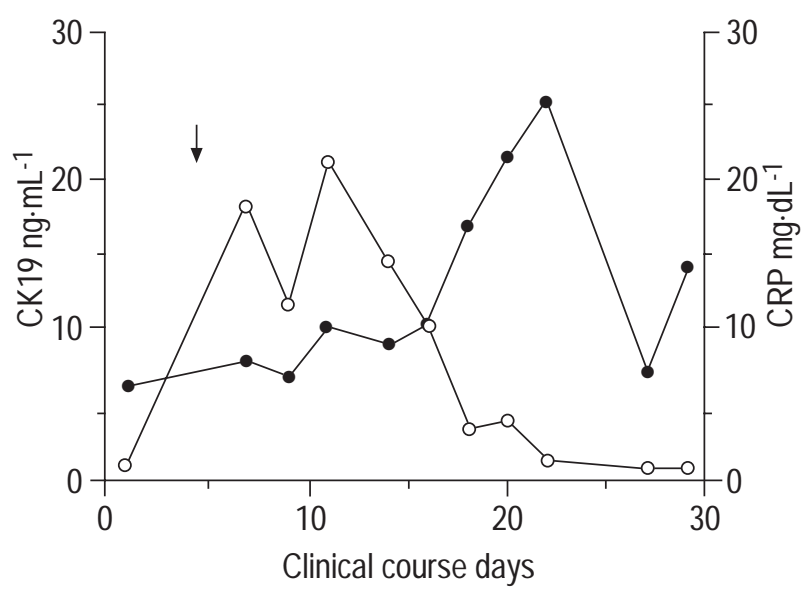

Fig. 4. - Changes in serum cytokeratin 19 fragment (CK19) levels (O) and $\mathrm{C}$-reactive protein (CRP) levels $(\mathrm{O})$ according to clinical courses in a patient with acute interstitial pneumonia (AIP). Arrow represents the onset of AIP. 
Table 1. - Correlations between cytokeratin 19 fragment (CK19) levels in serum and bronchoalveolar lavage fluid (BALF) with clinical parameters in patients with pulmonary fibrosis $(n=10)$

\begin{tabular}{llcccc}
\hline & Parameters & $\mathrm{R}$ & $\mathrm{p}$-value & \multicolumn{2}{c}{ 95\% CI } \\
\hline Serum & VC \% & -0.55 & 0.0165 & -0.809 & -0.112 \\
BALF & Macrophages \% & -0.811 & 0.0115 & -0.964 & -0.248 \\
& Neutrophils \% & 0.739 & 0.0341 & 0.071 & 0.949 \\
& Lymphocytes \% & 0.735 & 0.0358 & 0.062 & 0.948 \\
& Eosinophils \% & 0.869 & 0.0029 & 0.425 & 0.976 \\
& Lymphocytes* n & 0.907 & 0.0007 & 0.56 & 0.983 \\
& Eosinophils n & 0.979 & $<0.0001$ & 0.884 & 0.996 \\
BALF/albumin $^{+}$ & LDH in BALF & 0.838 & 0.0152 & 0.229 & 0.975 \\
& Neutrophils \% & 0.725 & 0.0402 & 0.041 & 0.946 \\
& Eosinophils \% & 0.79 & 0.0165 & 0.194 & 0.96 \\
& Eosinophils n & 0.815 & 0.0106 & 0.26 & 0.965 \\
& Albumin in BALF & -0.686 & 0.0261 & -0.919 & -0.1 \\
& CA19-9 in BALF & 0.697 & 0.0226 & 0.12 & 0.922 \\
\hline
\end{tabular}

*: Total number of lymphocytes ${ }^{+}$: CK19 values in BALF corrected to albumin. LDH: lactate dehydrogenase; CA19-9: carbohydrate antigen 19-9; CI: confidence interval; R: correlation coefficient.

Cytokeratin is a cytoskeletal structure expressed only in epithelial cells [1-4]. Twenty-seven subtypes of cytokeratin are known. Of these cytokeratins, CK19 is expressed in simple epithelia, including bronchial epithelial cells [1-4]. CK19 levels in serum have already been documented as a useful tumour marker for lung cancer [5-8]. However, there have been few studies which evaluate the clinical significance of CK19 in the sera and BALF of patients with pulmonary fibrosis.

IYONAGA et al. [13] reported that CK19 is expressed in hyperplastic type II pneumocytes, squamous metaplastic cells, bronchiolar metaplastic cells, and cuboidal metaplastic cells in patients with IPF.

NAKAMURA et al. [11] demonstrated that BALF from patients with chronic airway inflammatory disease contained higher levels of neutrophils and neutrophil elastase activity than BALF from nonsmoking controls subjects, and that the number of neutrophils in the BALF significantly correlated with the levels of CK19 in BALF. They also demonstrated that CK19 is released from bronchial epithelium by neutrophil-derived injurious substances.

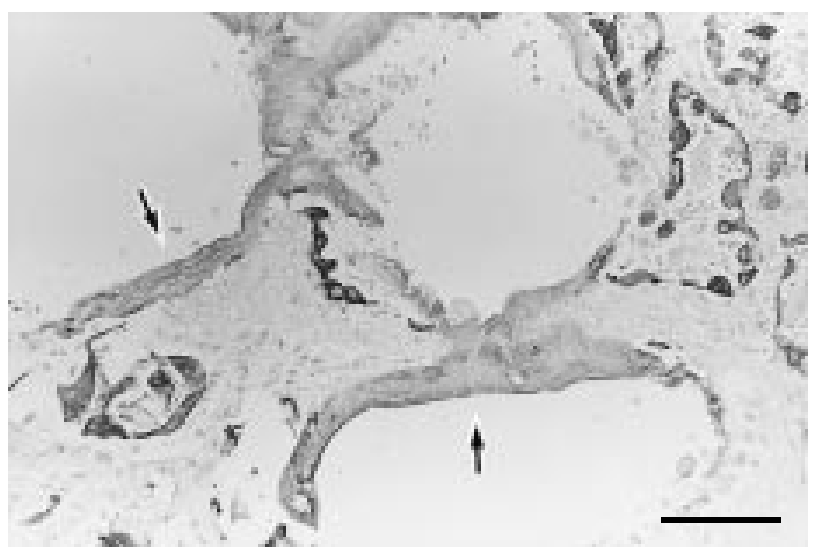

Fig. 5 - Immunohistochemical staining with anti-human cytokeratin 19 monoclonal antibody in a patient with acute interstitial pneumonia (AIP) showing a positive staining of the hyaline membrane (arrows). Note the strong positive reaction in the cytoplasm of proliferating type II pneumocytes. Staining was performed by the avidin-biotin-peroxidase complex method. Internal scale bar $=100 \mu \mathrm{m}$.
The present study demonstrated correlations between CK19 levels in serum and \%VC. In addition, CK19 in serum markedly increased in patients with AIP, and the serum CK19 levels were a useful marker to monitor the clinical course of acute lung injury. Furthermore, the presence of CK19 or its degraded products were clearly demonstrated in the hyaline membranes, and increased expression of CK19 was observed in proliferating type II pneumocytes. These data suggested that the CK19 levels in serum reflected the disease activity in patients with pulmonary fibrosis as well as AIP, and that CK19 may be a useful marker to prove the existence of the epithelial cell damage.

Successive measurements of serum CK19 and CRP in one patient showed an increase in CK19, but a decrease in CRP during therapy. CRP is produced from hepatocytes by the stimulation of interleukin (IL)-1, IL-6, and tumour necrosis factor (TNF)- $\alpha$. Since these cytokines are suppressed by corticosteroids, CRP decreased immediately after the steroid treatment. Epithelial cell damage as well as successive repair by alveolar type II cells may reflect the increase in CK19, explaining the discrepancy between the CRP values and CK19 values.

Interestingly, it was also demonstrated that CK19 in BALF correlated with the total number of both lymphocytes and eosinophils. IPF and PF-CVD are chronic inflammatory lung diseases of unknown aetiology which are characterized by the accumulation of neutrophils, macrophages, lymphocytes, and/or eosinophils [14, 15]. The presence of a high number of inflammatory cells in patients with the highest levels of CK19 in BALF probably indicates that a high degree of inflammation (alveolitis intensity) is associated with an increased epithelial cell injury. The significance of CK19 as a prognostic factor in pulmonary fibrosis should be evaluated in future studies.

In conclusion, these data demonstrated that cytokeratin 19 fragments in serum are increased in patients with pulmonary fibrosis as well as acute interstitial pneumonia, and that cytokeratin 19 fragments in serum and bronchoalveolar lavage fluid correlated with various clinical parameters in pulmonary fibrosis. These results suggest that the elevation of cytokeratin 19 fragment seems to be a marker of lung epithelial cell damage and repair. 


\section{References}

1. Moll R, Franke WW, Schiller DL. The catalog of human cytokeratins: patterns of expression in normal epithelia, tumors and cultured cells. Cell 1982; 31: 11-24.

2. Fuchs E. Keratins as biochemical markers of epithelial differentiation. Trends Genet 1983; 4: 277-281.

3. Coulombe PA. The cellular biology of keratins: beginning a new era. Curr Opin Cell Biol 1993; 5: 17-29.

4. Steinert PM. Structure, function, and dynamics of keratin intermediate filaments. J Invest Dermatol 1993; 100: 729-734.

5. Pujol JL, Grenier J, Daures JP, Daver A, Pujol H, Michel FB. Serum fragment of cytokeratin subunit 19 measured by CYFRA 21-1 immunoradiometric assay as a marker of lung cancer. Cancer Res 1993; 53: 61-66.

6. Muraki M, Tohda Y, Iwanaga T, Uejima H, Nagasaka Y, Nakajima S. Assessment of serum CYFRA 21-1 in lung cancer. Cancer 1996; 77: 1274-1277.

7. Lai RS, Hsu HK, Lu JY, Ger LP, Lai NS. CYFRA 21-1 enzyme-linked immunosorbent assay. Evaluation as a tumor marker in non-small cell lung cancer. Chest 1996; 109: 995-1000.

8. Pujol JL, Grenier J, Parrat E, et al. Cytokeratins as serum markers in lung cancer: A comparison of CYFRA 21-1 and TPS. Am J Respir Crit Care Med 1996; 154: 725-733.

9. Hirakata Y, Kobayashi J, Sugama Y, Kitamura S. Elevation of tumour markers in serum and bronchoalveolar lavage fluid in pulmonary alveolar proteinosis. Eur Respir $J$ 1995; 8: 689-696.

10. Suzuki A, Masuda T, Koito N, et al. Studies of serum markers in patients with interstitial pneumonia/pulmonary fibrosis complicated with collagen diseases: clinical evaluation of CYFRA21-1. Ryumachi 1996; 36: 837-843.

11. Nakamura H, Abe S, Shibata $\mathrm{Y}$, et al. Elevated levels of cytokeratin 19 in the bronchoalveolar lavage fluid in patients with chronic airway inflammatory disease. A specific marker for bronchial epithelial injury. $\mathrm{Am} \mathrm{J}$ Respir Crit Care Med 1997; 155: 1217-1221.

12. Katzenstein ALA, Myers JL, Mazur MT. Acute interstitial pneumonia: a clinicopathologic, ultrastructural, and cell kinetic study. Am J Surg Pathol 1986; 10: 256-267.

13. Iyonaga K, Miyafuji M, Suga M, Saita N, Ando M. Alterations in cytokeratin expression by the alveolar lining epithelial cells in lung tissue from patients with idiopathic pulmonary fibrosis. $J$ Pathol 1997; 182: 217 224.

14. Crystal RG, Bitterman PB, Rennard SI, Hance AJ, Keogh BA. Interstitial lung diseases of unknown cause. Disorders characterized by chronic inflammation of the lower respiratory tract. N Engl J Med 1984; 310: 154166.

15. Peterson MW, Monick M, Hunninghake GW. Prognostic role of eosinophils in pulmonary fibrosis. Chest 1987; 92: $51-56$. 